\title{
OSTEOBLASTS GENERATE AN OSTEOGENIC MICROENVIRONMENT WHEN GROWN ON SURFACES WITH ROUGH MICROTOPOGRAPHIES
}

\author{
B.D. Boyan ${ }^{1 *}$, S. Lossdörfer ${ }^{2}$, L. Wang ${ }^{1}$, G. Zhao ${ }^{1}$, C.H. Lohmann ${ }^{3}$, D.L. Cochran ${ }^{4}$ and Z. Schwartz ${ }^{1,4,5}$
}

${ }^{1}$ Department of Biomedical Engineering, Georgia Institute of Technology, Atlanta, Georgia, USA, ${ }^{2}$ Department of Orthodontics, University of Bonn, Bonn, Germany, ${ }^{3}$ Department of Orthopaedics, University of HamburgEppendorf, Hamburg, Germany, ${ }^{4}$ Department of Periodontics, University of Texas Health Science Center at San Antonio, San Antonio, Texas, USA, ${ }^{5}$ Department of Periodontics, Hebrew University Hadassah, Jerusalem, Israel

\begin{abstract}
Osteoblasts respond to microarchitectural features of their substrate. On smooth surfaces (tissue culture plastic, tissue culture glass, and titanium), the cells attach and proliferate but they exhibit relatively low expression of differentiation markers in monolayer cultures, even when confluent. When grown on microrough Ti surfaces with an average roughness $(\mathrm{Ra})$ of 4-7 $\mu \mathrm{m}$, proliferation is reduced but differentiation is enhanced and in some cases, is synergistic with the effects of surface microtopography. In addition, cells on microrough Ti substrates form hydroxyapatite in a manner that is more typical of bone than do cells cultured on smooth surfaces. Osteoblasts also respond to growth factors and cytokines in a surface-dependent manner. On rougher surfaces, the effects of regulatory factors like $1 \alpha, 25(\mathrm{OH})_{2} \mathrm{D}_{3}$ or $17 \beta$-estradiol are enhanced. The response to the surface is mediated by integrins, which signal to the cell through many of the same mechanisms used by growth factors and hormones. Studies using PEGmodified surfaces indicate that increased differentiation may be related to altered attachment to the surface. When osteoblasts are grown on surfaces with chemistries or microarchitectures that reduce cell attachment and proliferation, and enhance differentiation, the cells tend to increase production of factors like TGF- $\beta 1$ that promote osteogenesis while decreasing osteoclastic activity. Thus, on microrough $\mathrm{Ti}$ surface, osteoblasts create a microenvironment conducive to new bone formation.
\end{abstract}

Key Words: Osteoblasts, titanium, surfaces, microtopography, osteoprotegerin (OPG), osteogenesis.

\footnotetext{
*Address for correspondence:

Barbara D. Boyan

Wallace H. Coulter Department of Biomedical Engineering at Georgia Tech and Emory University, Georgia Institute of Technology

315 Ferst Drive NW, Atlanta, Georgia 30332

Telephone Number: 404-385-4108

FAX Number: 404-894-2291

E-mail: Barbara.Boyan@,bme.gatech.edu
}

\section{Introduction}

Cell attachment to materials and cell proliferation are sensitive to the microtopography of the surface (see Boyan et al., 1995 for a review). The present paper reviews work from our laboratory showing that extracellular matrix synthesis and differentiation of osteoblasts are sensitive to surface microtopography as well. On titanium surfaces with microrough rugosities, osteoblasts assume a more differentiated morphology. Attachment is reduced on these surfaces in comparison with tissue culture plastic or smooth titanium surfaces (Lohmann et al., 2000). In addition, those cells that do attach exhibit reduced proliferation. In contrast, these cells show increased markers of osteoblastic differentiation, including changes in matrix vesicle alkaline phosphatase specific activity and osteocalcin production (Martin et al., 1996).

These observations suggest that structural features of the surface modulate osteogenesis. Studies examining the response of MG63 osteoblast-like human osteosarcoma cells to dentine and bone surfaces support this observation (Schwartz et al., 2000; Boyan et al., 2003). When these cells are cultured on dentin or bone wafers, they exhibit reduced proliferation and increased differentiation in comparison with cells grown on tissue culture plastic. This surface effect is further enhanced when the wafers are treated with osteoclasts. It is clear that part of the effect of osteoclastic resorption results from conditioning of the surface and not simply from removal of mineral. If only $3 \%$ of the dentine surface is removed via osteoclastic resorption, the effect on osteoblasts is greater than that caused by demineralization of the entire surface with tetracycline. Others have shown that osteoclasts deposit attachment factors during the resorptive process (Sheu et al., 2003). However, at least part of the osteoclastic response is microtopography-dependent. Resorption of mineral leaves a complex micro and nanotopography that can be modeled using titanium substrates. Recent experiments from our laboratory using these models are summarized below.

\section{The Model System}

To understand how microtopography modulates cell response, human osteoblast-like cells are cultured on titanium surfaces with three different surface designs. Smooth surfaces have an average roughness ( $\mathrm{Ra}$ ) of $<0.6$ $\mu \mathrm{m}$. These surfaces are then grit blasted followed by an acid etch, resulting in craters between 30 and $100 \mu \mathrm{m}$ in diameter that are covered with pits between 1 to $3 \mu \mathrm{m}$ in diameter; this surface has an overall $\mathrm{Ra}$ of $4-5 \mu \mathrm{m}$. Al- 


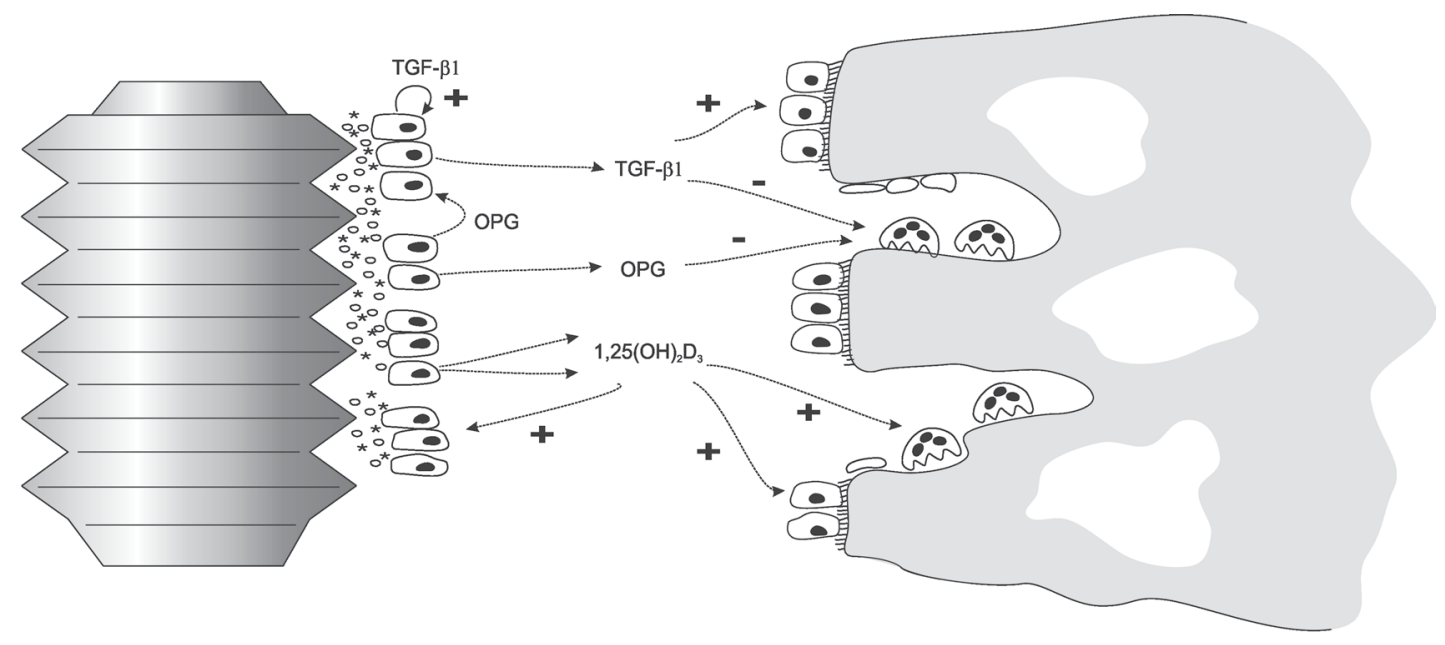

Figure 1. Schematic diagram showing the effects of rough microtopography on production of paracrine factors by osteoblasts during peri-implant bone formation. Osteoblasts synthesize osteoid on the implant surface as well as on the normal bone surface. Levels of latent TGF- $\beta 1$ are increased in the extracellular matrix, as well as in the extracellular fluid. Once activated, the growth factor can stimulate osteoblast proliferation, extracellular matrix synthesis and alkaline phosphatase activity $(+)$. At the same time, active TGF- $\beta 1$ inhibits osteoclastic activity (-). Osteoblasts also increase levels of the decoy receptor osteoprotegerin (OPG), which binds RANK ligand (RANKL) on the surface of the osteoblast, as well as any soluble RANKL, thereby preventing osteoclast formation and activation due to RANKL binding to its receptor (RANK) on the osteoclast. Soluble RANKL is not detectable in cultures of osteoblasts grown on Ti surfaces with rough microtopographies and RANKL mRNA levels are low, so these components are not included in the scheme. Osteoblasts also produce elevated levels of 1,25 dihydroxyvitamin $\mathrm{D}_{3}\left(1,25(\mathrm{OH})_{2} \mathrm{D}_{3}\right)$ on rough surfaces. $1,25(\mathrm{OH})_{2} \mathrm{D}_{3}$ promotes osteoclast formation due to fusion of monocytes and acts on osteoblasts promoting their differentiation. $1 \alpha, 25(\mathrm{OH})_{2} \mathrm{D}_{3}$ also stimulates matrix calcification through rapid activation of $\mathrm{Ca}^{2+}$ ion deposition.

ternatively the smooth surface is coated with irregular projections of Ti created via titanium plasma spray, with an $\mathrm{Ra}$ of $>5 \mu \mathrm{m}$. All surfaces are prepared as disks to fit the well of a 24-well culture plate. In each experiment, six individual cultures are examined per variable. Cells grown on tissue culture plastic serve as controls and in some experiments cells grown on tissue culture glass are also included. Two different cell culture models are used for the studies described here. MG63 cells are human osteoblast-like osteosarcoma cells and have a phenotype of a relatively immature osteoblast. These cells are cultured in Dulbecco's modified Eagle medium (DMEM) in the presence of $10 \%$ fetal bovine serum (FBS) and 1\% antibiotics. For experiments examining the effects of substrate microtopography on response to regulatory factors, confluent MG63 cells are treated for an additional 24 hours with media containing vehicle or the agent of interest. NHOst osteoblast-like cells are provided by a commercial company and are derived from normal human bone biopsies. The NHOst cells used in our studies were from an adult female. These cells were cultured in osteoblast growth media (OGM) purchased from Clonetics (San Diego, CA). All experiments are repeated to ensure validity of the observations. Data are analyzed by analysis of variance and specific differences between groups were determined by the Bonferroni modification of Student's t-test.

\section{Rough Surfaces Promote Osteogenesis}

In vivo, a material surface is coated by components present in the extracellular fluid. This process is sensitive to surface chemistry. In addition, the structural features of the surface regulate adsorption via alterations in free energy. The first cells to interact with the material are neutrophils and macrophages (Davies, 2003), and it is likely that they further modify the adsorption layer on the surface through the release of cytokines and inflammatory mediators. In cell culture, using serum-containing medium approximates this. Using this system, we have shown that osteoblasts can attach, proliferate and differentiate into secretory osteoblasts that exhibit increased levels of alkaline phosphatase specific activity and osteocalcin (Martin et al., 1996). These cells also produce factors that are known to modulate osteoclastic activity as well as osteoblastic activity, as described below (Fig. 1). Moreover, osteoblast phenotypic expression and the levels of regulatory factors in the conditioned media are modulated by $1 \alpha, 25(\mathrm{OH})_{2} \mathrm{D}_{3}$ in a manner that is synergistic with surface roughness (Boyan et al., 1998). Others have shown that response to BMP-2 depends on surface features (Ong et al., 1997) and we have shown that fetal rat calvarial cells are regulated by BMP-2 and dexamethasone under similar culture conditions (Boyan et al., 2002). Moreover, normal human osteoblasts are regulated by $17 \beta$-estradiol in a roughness dependent manner (Lohmann et al., 2002).

Many of the factors that are produced by the cells in increased amounts are known to regulate bone remodeling by differentially affecting osteoblasts and osteoclasts. As surface microroughness increases, levels of $\mathrm{PGE}_{1}$ and 
Total TGF-B1

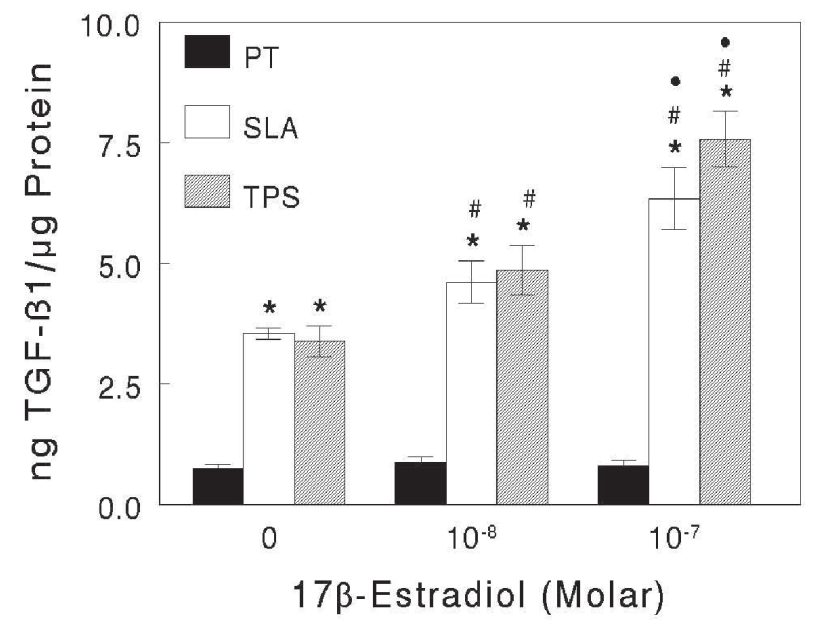

Figure 2. Effect of surface microtopography on TGF$\beta 1$ levels in the conditioned media of normal female human osteoblasts (NHOst cells) cultured on smooth Ti (PT), Ti with a moderately rough microarchitecture achieved by grit blasting and acid etching (SLA), and Ti with a rough microarchitecture achieved by Ti plasma spray (TPS). Confluent cultures of NHOst cells were treated with control media or media containing $10^{-8}$ or $10^{-7} \mathrm{M} 17 \beta$-estradiol. Total TGF- $\beta 1$ in the conditioned media was measured using an immunoassay kit. Data were normalized to protein content of the cell layer, but similar results were obtained when data were normalized to cell number. Values are means \pm SEM for 6 independent cultures per variable. Data were analyzed by ANOVA and statistical differences between groups determined using the Bonferroni modification of Student's t-test. All experiments were repeated at least twice to ensure validity of the observations. Data shown are from one representative experiment. $* \mathrm{p}<0.05$, v. smooth (PT) v. rough (SLA or TPS) for each concentration of $17 \beta$-estradiol; $\# \mathrm{p}<0.05$, with $17 \beta$-estradiol v. no hormone on each surface.

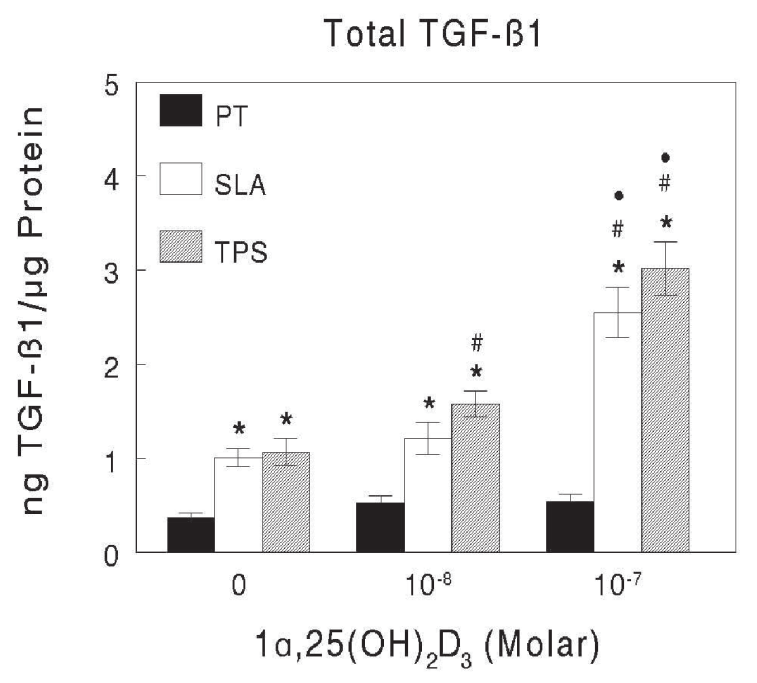

Figure 3. Effect of surface microtopography on TGF- $\beta 1$ levels in the conditioned media of MG63 cells cultured on smooth Ti (PT), Ti with a moderately rough microarchitecture achieved by grit blasting and acid etching (SLA), and Ti with a rough microarchitecture achieved by Ti plasma spray (TPS). Confluent cultures of MG63 human osteoblast-like cells were treated with control media or media containing $10^{-8}$ or $10^{-7} \mathrm{M} 1 \alpha, 25(\mathrm{OH})_{2} \mathrm{D}_{3}$. Total TGF- $\beta 1$ in the conditioned media was measured using an immunoassay kit. Data were normalized to protein content of the cell layer, but similar results were obtained when data were normalized to cell number. Values are means \pm standard error of the mean (SEM) for 6 independent cultures per variable. Data were analyzed by ANOVA and statistical differences between groups determined using the Bonferroni modification of Student's t-test. All experiments were repeated at least twice to ensure validity of the observations. Data shown are from one representative experiment. ${ }^{*} \mathrm{p}<0.05$, v. smooth (PT) v. rough (SLA or TPS) for each concentration of $1 \alpha, 25(\mathrm{OH})_{2} \mathrm{D}_{3}$; $\# \mathrm{p}<0.05$, with $1 \alpha, 25(\mathrm{OH})_{2} \mathrm{D}_{3}$ v. no hormone on each surface.
$\mathrm{PGE}_{2}$ in the conditioned medium also increase (Kieswetter et al., 1996). $\mathrm{PGE}_{2}$ stimulates osteoclastic activity at high levels, but it is required at low levels for osteoblast activity (Raisz 1984; Raisz 1990). The elevated prostaglandins that are seen in cultures grown on rough microtopographies appear to be required for the enhanced osteogenesis since inhibition of prostaglandin production by indomethacin blocks the increase in osteoblast phenotypic expression on these substrates (Sisk et al., 2001). When MG63 cells are grown on tissue culture plastic or smooth Ti surfaces, $1 \alpha, 25(\mathrm{OH})_{2} \mathrm{D}_{3}$ has little or no effect on $\mathrm{PGE}_{2}$ production. $1 \alpha, 25(\mathrm{OH})_{2} \mathrm{D}_{3}$ stimulates $\mathrm{PGE}_{2}$ production only when MG63 cells are grown on the rougher surfaces and this stimulatory effect is dose-dependent and synergistic with the surface effect (Boyan et al., 1998). Similarly 17ß-estradiol causes an increase in $\mathrm{PGE}_{2}$ production by normal human osteoblasts that is synergistic with surface microtopography (Lohmann et al., 2002).
TGF- $\beta 1$ levels are also surface-dependent (Kieswetter et al., 1996). MG63 cells produce low levels of this growth factor when cultured on tissue culture plastic and smooth Ti surfaces. However, when these cells are grown on microrough surfaces, constitutive production is increased. The amount of TGF- $\beta 1$ produced by osteoblasts cultured on Ti is also modulated in a surface-dependent manner by factors that regulate osteogenesis and subsequent bone resorption. 17 $\beta$-estradiol has no effect on TGF- $\beta 1$ content of the media of normal female human osteoblasts (NHOst cells) grown on smooth Ti surfaces but when NHOst cells are cultured on rougher microtopographies, $17 \beta$-estradiol causes a dose-dependent increase in the levels of TGF- $\beta 1$ (Fig. 2) (Lohmann et al., 2002). $1 \alpha, 25(\mathrm{OH})_{2} \mathrm{D}_{3}$ causes a similar effect in MG63 osteoblast-like cells (Boyan et al., 1998). TGF- $\beta 1$ levels in cultures grown on smooth $\mathrm{Ti}$ are not affected, but $1 \alpha, 25(\mathrm{OH})_{2} \mathrm{D}_{3}$ increases TGF- $\beta 1$ 
levels in the media of cells grown on rougher surfaces and the effect is dose-dependent (Fig. 3). In addition, the effect is targeted to TGF- $\beta 1$ that is incorporated into the cell layer (Schwartz et al., 2001a). Thus, the behavior of cells grown on surfaces with rough microarchitectures is altered both with respect to their basal production of autocrine factors and with respect to their ability to produce these factors in response to hormonal regulation. Moreover, the response is to increase production of a factor that promotes early stages of bone formation and retards osteoclastic resorption.

Regulation of TGF- $\beta 1$ is important to bone formation for a number of reasons. This growth factor stimulates proliferation of mesenchymal cells and enhances the production of extracellular matrix, particularly of type I collagen. TGF- $\beta 1$ is synthesized in latent form and stored in the extracellular matrix together with a binding protein. When MG63 cells are cultured on Ti surfaces of increasing roughness, most of the TGF- $\beta 1$ produced is in latent form, and the amount increases on surfaces with rough microtopographies (Kieswetter et al., 1996). In addition, the distribution of TGF- $\beta 1$ between the matrix and the conditioned media of the cells is affected as well. On rougher surfaces, there is a greater incorporation of TGF $\beta 1$ into the matrix (Schwartz et al., 2001a). This is important for later bone remodeling because osteoclasts release TGF- $\beta 1$ during the resorption phase and activate it. Once activated, TGF- $\beta 1$ can act on the osteoclast to down regulate its activity.

These studies suggested to us that other factors that modulate osteoclastogenesis might be modulated as well. Osteoblasts modulate osteoclasts via the RANK ligand (RANKL) regulatory system recently described in a review by Khosla (Khosla, 2001). Osteoblasts retain RANKL on their plasma membranes. When RANKL binds it receptor RANK on osteoclasts, osteoclastic resorption is activated. To control this phenomenon, osteoblasts also produce a decoy receptor termed osteoprotegerin (OPG), which binds to RANKL, preventing it from interacting with RANK. If the regulatory stimulus now favors osteoclasts but excess OPG is present, the osteoblast may also produce soluble RANKL (sRANKL), which can then bind the OPG, leaving membrane associated RANKL to interact with RANK on the osteoclasts. Thus, excess OPG would favor osteogenesis over bone resorption. Our preliminary results, presented at the 2003 International Association for Dental Research meeting in Gothenburg, Sweden, indicate that this is indeed the case. When grown on rougher microtopographies, MG63 cells produce higher levels of OPG and this is further increased by $1 \alpha, 25(\mathrm{OH})_{2} \mathrm{D}_{3}$ in a synergistic manner, but no change occurs in the expression of sRANKL.

\section{Mechanisms Mediating the Microtopography Effect}

Prostaglandin plays an important role in mediating the effects of surface microtopography on osteoblast physiology. As noted above, the amount of $\mathrm{PGE}_{1}$ and $\mathrm{PGE}_{2}$ released into the conditioned media increases as surface microroughness increases (Kieswetter et al., 1996). Block- ing prostaglandin production with the general cyclooxygenase (Cox) inhibitor indomethacin, also blocks the increase in markers typical of mature osteoblasts. Both constitutive Cox-1 and inducible Cox-2 are involved, as inhibitors specific to only one form of the enzyme fail to block all prostaglandin-dependent responses (Boyan et al., 2001).

Cells that are attachment dependent interact with their substrate through a number of mechanisms including specific binding of integrins to components of the extracellular matrix, as well as to proteins adsorbed on the substrate surface. Others have shown that one of the integrins that mediate bone function and binding of osteoblasts to proteins containing the arginine-glycineaspartic acid (RGD) motif is $\alpha_{5}, \beta_{1}$ (Schaffner and Dard, 2003). This binding initiates a signaling cascade resulting in new gene expression and protein synthesis. The signaling cascades initiated by integrin binding include protein kinase $\mathrm{C}$ (PKC)-dependent phosphorylation, culminating in mitogen activated protein (MAP) kinase activation. Part of the effect of $1 \alpha, 25(\mathrm{OH})_{2} \mathrm{D}_{3}$ on osteoblasts is also mediated via this signaling pathway (Schwartz et al., 2001b), which may explain why MG63 cells respond in a synergistic manner to this vitamin D metabolite when they are cultured on microrough topographies. For these studies, confluent cultures of MG63 cells were cultured for 24 hours on smooth $(\mathrm{Ra}<0.6 \mu \mathrm{m})$ and rough $(\mathrm{Ra} 4-5$ $\mu \mathrm{m})$ titanium disk surfaces in the presence and absence of $10^{-8} \mathrm{M} 1 \alpha, 25(\mathrm{OH})_{2} \mathrm{D}_{3}$. One half of the cultures were treated with the MAP kinase inhibitor PD98059, which is specific for the ERK1/2 family of MAP kinases. The results of this study show that the decrease in cell number and increase in osteocalcin levels due to surface roughness are not regulated in this manner although the enhanced response to $1 \alpha, 25(\mathrm{OH})_{2} \mathrm{D}_{3}$ is via ERK $1 / 2$. In contrast, the increase in alkaline phosphatase specific activity on rough surfaces is due in part to ERK1/2, as is the stimulatory effect of $1 \alpha, 25(\mathrm{OH})_{2} \mathrm{D}_{3}$ on this enzyme activity. Thus, multiple interacting pathways may modulate phenotypic expression due to surface microtopography.

Osteoblasts assume distinct morphologies depending on the architectural features of their substrate. On microrough surfaces, as long as the peak-to-peak distance is less than the length of the cell body (Brunette, 1988), the cell bodies become more cuboidal, and anchor themselves to the surface through long dendritic filopodia (Brunette, 1986). In contrast on the smoother surfaces, the cells flatten and spread, resulting in a fibroblastic appearance. The cell morphology correlates with the physiological behaviour of the cells. On smooth surfaces, prostaglandin synthesis is low, TGF- $\beta 1$ levels are low, alkaline phosphatase specific activity is low, and osteocalcin levels are low, whereas proliferation rates are relatively high in comparison with cells cultured on rougher surfaces (Kieswetter et al., 1996). Moreover, these parameters are either not affected by $1 \alpha, 25(\mathrm{OH})_{2} \mathrm{D}_{3}$ or the effect of the hormone is minimal, supporting a fibroblastic phenotype. In contrast, on rougher surfaces, proliferation is reduced and the other parameters are increased, including responsiveness to $1 \alpha, 25(\mathrm{OH})_{2} \mathrm{D}_{3}$, supporting a mature secretory 
osteoblast-like phenotype (Boyan et al., 1998).

It is not yet clear if the change in morphology precedes the expression of a more mature physiology or if the differentiation of the cell is activated by another yet unidentified factor and the cell now responds differently to the surface microarchitecture. Our observations suggest that the architecture of the surface forces the cells to adapt their morphology and this is translated to the cell through the integrin receptor system. To test this, in collaboration with Marcus Textor and Samuele Tosatti at the ETH (Zürich, Switzerland), we cultured MG63 cells on smooth and microrough Ti disks that were coated with polyethylene glycol (PEG) grafted to poly-L-lysine (PLLg-PEG) to limit nonspecific adhesion of serum proteins. The experiment was conducted in three formats: (A) cells were cultured on PLL-g-PEG alone, PLL-g-PEG on which a subset of PEG was modified to exhibit an RGD-peptide, and PLL-g-PEG on which a subset was modified to exhibit an RDG-peptide; (B) PLL-g-PEG surfaces containing varying concentrations of the RGD-peptide up to the concentration tested under format A; and (C) PLL-g-PEG as defined in subset $\mathrm{A}$ in the presence of a soluble competing RGD peptide. The results of this study (Tosatti et al., 2003) indicate that MG63 cells will attach to PLL-gPEG and those that do so exhibit the physiology of a mature osteoblast. Perhaps the most important observation is that inclusion of the RGD peptide on the surface restores the cells to the immature phenotype noted on smooth surfaces and tissue culture plastic, whereas inclusion of the inactive RDG peptide has no effect.

Responses to the surface also depend on the state of maturation of the cell in the osteoblast lineage. We have examined numerous cell lines and primary cell cultures in this model, from the multipotent fetal rat calvarial cells to the osteocyte cell line MLO-Y4 (Boyan et al., 2002; Lohmann et al., 2000). These experiments indicate that as cells become more mature, the stimulatory effect of the microrough surface on differentiation becomes attenuated. However, it is only on rough surfaces and only in the presence of BMP-2, that fetal rat calvarial cells are able to establish three-dimensional nodules that form mineral in a physiologically relevant manner, characterized by poorly crystalline hydroxyapatite deposited in association with extracellular matrix collagen (Boyan et al., 2002). Why this is the case is not yet clear. The results support in vivo observations that a material can affect cells directly on the surface as well as cells distal to the biomaterial (Braun et al., 1995) indicating that the extracellular signaling factors released by the cells in direct contact with the material are sensed by other cells in the microenvironment, and potentially systemically as well.

\section{Summary}

Our laboratory has developed a well-defined in vitro model to examine the interaction of cells and materials, focusing on the role that microrough architectural features play in modulating osteoblast behaviour. These studies show that cells respond to surface microtopography in a cell maturation dependent manner. In addition, the surface can alter the maturation state of the cell, such that it releases signaling factors typical of its new maturation state and that it responds to exogenous regulatory factors in a manner typical of its new maturation state. Because integrins and many of these factors use interacting signaling pathways, potential for synergy is increased.

Our results also demonstrate the potential to misinterpret the value of various surface features to cell response in vitro and in vivo if one examines only cell attachment and proliferation without considering other physiological parameters. Osteogenesis in vivo must find a balance between achieving adequate coverage of an implant material with osteogenic cells and the ability of those cells to differentiate into competent osteoblasts in a timely manner. Materials that support only attachment and spreading are likely to favour connective tissue interfaces rather than bone.

\section{Acknowledgements}

The authors thank the students and staff that have contributed to these studies over the years. This paper was not constructed as a literature review but as a summary of work in our laboratory. We are particularly indebted to our collaborators in Switzerland at the ETH (Zürich), Professor Marcus Textor and Dr. Samuele Tosatti, and at the Institut Straumann (Waldenburg), Drs. James Simpson, Alain Denzer, and Marco Wieland. We thank the Institut Straumann for fabricating the titanium disks for our studies and the ITI Foundation (Waldenburg, Switzerland) for their support of our work. Partial support was also provided via the Georgia Tech/Emory Center for the Engineering of Living Tissues, Georgia Institute of Technology, Atlanta, Georgia and the Georgia Research Alliance.

\section{References}

Boyan BD, Hummert TW, Kieswetter K, Schraub D, Dean DD, Schwartz Z (1995) Effect of titanium surface characteristics on chondrocytes and osteoblasts in vitro. Cells Mater 5: 323-335.

Boyan BD, Batzer R, Kieswetter K, Liu Y, Cochran DL, Szmuckler-Moncler S, Dean DD, Schwartz Z (1998) Titanium surface roughness alters responsiveness of MG63 osteoblast-like cells to $1 \alpha, 25-(\mathrm{OH})_{2} \mathrm{D}_{3}$. J Biomed Mater Res 39: 77-85.

Boyan BD, Lohmann CH, Sisk M, Liu Y, Sylvia VL, Cochran DL, Dean DD, Schwartz Z (2001) Both cyclooxygenase-1 and cyclooxygenase- 2 mediate osteoblast response to titanium surface roughness. J Biomed Mater Res 55: 350-359.

Boyan BD, Bonewald LF, Paschalis EP, Lohmann CH, Rosser J, Cochran DL, Dean DD, Schwartz Z, Boskey AL (2002) Osteoblast-mediated mineral deposition in culture is dependent on surface microtopography. Calcif Tissue Int 71: 519-529.

Boyan BD, Schwartz Z, Lohmann CH, Sylvia VL, Cochran DL, Dean DD, Puzas JE (2003) Pretreatment of 
bone with osteoclasts affects osteoblast phenotypic expression. J Orthop Res 21: 638-647.

Braun G, Kohavi D, Amir D, Luna MH, Caloss R, Sela J, Dean DD, Boyan BD, Schwartz Z (1995) Markers of primary mineralization are correlated with bone-bonding ability of titanium or stainless steel in vivo. Clin Oral Implants Res 6: 1-13.

Brunette DM (1986) Spreading and orientation of epithelial cells on grooved substrata, Exp Cell Res 167: 203-217.

Brunette DM (1988) The effects of implant surface topography on the behavior of cells, Int J Oral Maxillofac Implants 3: 231-246.

Davies JE (2003) Understanding peri-implant endosseous healing. J Dent Educ 67: 932-949.

Kieswetter K, Schwartz Z, Hummert TW, Cochran DL, Simpson J, Dean DD, Boyan BD (1996) Surface roughness modulates the local production of growth factors and cytokines by osteoblast-like MG63 cells. J Biomed Mater Res 32: 55-63.

Khosla S (2001) Minireview: the OPG/RANKL/ RANK system. Endocrinology 142: 5050-5055.

Lohmann CH, Bonewald LF, Sisk MA, Sylvia VL, Cochran DL, Dean DD, Boyan BD, Schwartz Z (2000) Maturation state determines the response of osteogenic cells to surface roughness and 1,25-dihydroxyvitamin $\mathrm{D}_{3}$. J Bone Miner Res 15: 1169-1180.

Lohmann CH, Tandy EM, Sylvia VL, Hell-Vocke AK, Cochran DL, Dean DD, Boyan BD, Schwartz Z (2002) Response of normal female human osteoblasts (NHOst) to $17 \beta$-estradiol is modulated by implant surface morphology. J Biomed Mater Res 62: 204-213.

Martin JY, Dean DD, Cochran DL, Simpson J, Boyan BD, Schwartz Z (1996) Proliferation, differentiation, and protein synthesis of human osteoblast-like cells (MG63) cultured on previously used titanium surfaces. Clin Oral Impl Res 7: 27-37.

Ong JL, Cardenas HL, Cavin R, Carnes DL Jr (1997) Osteoblast responses to BMP-2-treated titanium in vitro. Int J Oral Maxillofac Impl 12: 649-654.
Raisz LG (1984) Studies on bone formation and resorption in vitro. Horm Res 20: 22-27.

Raisz LG (1990) The role of prostaglandins in the local regulation of bone. Prog Clin Biol Res 332: 195-203.

Schaffner P, Dard MM (2003) Structure and function of RGD peptides involved in bone biology. Cell Mol Life Sci 60: 119-132.

Schwartz Z, Lohmann CH, Wieland M, Cochran DL, Dean DD, Textor M, Bonewald LF, Boyan BD (2000) Osteoblast proliferation and differentiation on dentin slices are modulated by pretreatment of the surface with tetracycline or osteoclasts. J Periodontol 71: 622-633.

Schwartz Z, Lohmann CH, Sisk M, Cochran DL, Sylvia VL, Simpson J, Dean DD, Boyan BD (2001a) Local factor production by MG63 osteoblast-like cells in response to surface roughness and $1,25-(\mathrm{OH})_{2} \mathrm{D}_{3}$ is mediated via protein kinase $\mathrm{C}$ - and protein kinase A-dependent pathways. Biomaterials 22: 731-741.

Schwartz Z, Lohmann CH, Vocke AK, Sylvia VL, Cochran DL, Dean DD, Boyan BD (2001b) Osteoblast response to titanium surface roughness and $1 \alpha, 25-(\mathrm{OH})_{2} \mathrm{D}_{3}$ is mediated through the mitogen activated protein (MAP) kinase pathway. J Biomed Mater Res 56: 417-426.

Sheu TJ, Schwarz EM, Martinez DA, O'Keefe RJ, Rosier RN, Zuscik MJ, Puzas JE (2003) A phage display technique identifies a novel regulator of cell differentiation. J Biol Chem 278: 438-443.

Sisk M, Lohmann CH, Cochran DL, Sylvia VL, Simpson JP, Dean DD, Boyan BD, Schwartz Z (2001) Inhibition of cyclooxygenase by indomethacin modulates osteoblast response to titanium surface roughness in a time-dependent manner. Clin Oral Implants Res 12: 5261.

Tosatti S, Schwartz Z, Campbell C, Cochran DL, VandeVondele S, Hubbell JA, Denzer A, Simpson J, Wieland M, Lohmann CH, Textor M, Boyan BD (2003) RGD-containing peptide GCRGYGRGDSPG reduces enhancement of osteoblast differentiation by poly(Llysine)-graft-poly(ethylene glycol) coated titanium surfaces. J Biomed Mat Res, in press 